1 Hacettepe Journal of Mathematics and Statistics

h Volume 47 (4) (2018), 805-812

\title{
A combinatorial approach to the classification of resolution graphs of weighted homogeneous plane curve singularities
}

\author{
Muhammad Ahsan Binyamin*, Hafiz Muhammad Afzal Siddiqui ${ }^{\dagger}$ and Amir \\ Shehzad $^{\ddagger}$
}

\begin{abstract}
In this article we describe the classification of the resolution graphs of weighted homogeneous plane curve singularities in terms of their weights by using the concepts of graph theory and combinatorics. The classification shows that the resolution graph of a weighted homogeneous plane curve singularity is always a caterpillar.
\end{abstract}

Keywords: Plane Curves, Weights, Caterpillar.

Mathematics Subject Classification (2010): 14Q05,14H20

Received: 21.11.2016 Accepted : 08.02.2017 Doi : 10.15672/HJMS.2017.460

\section{Introduction}

The history of resolution of plane curve singularities is very old. It started with Newton in 1676, who showed the existence of Puiseux series. The resolution of plane curve singularities is an easy consequence. There is a large group of mathematicians who introduced new methods to resolve a plane curve singularity and they found deep and important applications of resolution of plane curve singularities. János Kollár lists about 20 ways of resolution of plane curve singularities (cf. [5]). Moreover an algebraic and combinatorial information about plane curve singularities can be found in [7], [9].

A graph is an ordered pair $G=(V, E)$, where $V$ is called vertex set and $E$ is called edge set. $|V|$ and $|E|$ denote the order and the size of a graph, respectively. A tree is an acyclic connected graph with $n$ vertices and $n-1$ edges and a caterpillar is a special type of tree with the property that a path remains if all leaves are deleted. A vertex labeling is

\footnotetext{
*Department of Mathematics, Government College University, Faisalabad, Pakistan, Email : ahsanbanyamin@gmail.com

${ }^{\dagger}$ Department of Mathematics, COMSATS Institute of Information Technology Lahore, Pakistan,

Email : hmasiddiqui@gmail.com

‡Department of Mathematics, Government College University, Faisalabad, Pakistan, Email : zain.ahmad54@yahoo.com
} 
a bijection from the set of vertices $V$ to the set of labels $\{1,2, \ldots,|V|\}$.

In [2], S. Dale Cutkosky and H. Srinivasan compute the resolution graph combinatorically by using the characteristic pairs of an irreducible plane curve singularity. Also Y. Jingen in [4], associates each singularity of a curve on a surface to a tree called S-tree which is some kind of "structured graph" and obtained by using the procedure of blow-ups.

In this article we introduce a combinatorial approach to compute the resolution graph of a weighted homogeneous plane curve singularity. As a first step we compute the order and the size of the resolution graph of a weighted homogeneous plane curve singularity. Then we define a vertex labeling on the set of vertices obtained from the weights of a weighted homogeneous plane curve singularity and finally construct the resolution graph.

\section{Basic Definitions}

In this section, we give some basic definitions related to the resolution of plane curve singularities. Definitions can be found in [3].

2.1. Definition. Let $B l(0)_{\mathbb{C}^{2}}$ denote the blowing - up of $0 \in \mathbb{C}^{2}$, which is the subset of $\mathbb{C}^{2} \times P$, where $P$ is the projective line and it is defined as

$$
B l(0)_{\mathbb{C}^{2}}=\left\{\left(a, l_{a}\right): a \in l_{a}\right\},
$$

where $a$ is a point and $l_{a}$ is a line in $\mathbb{C}^{2}$ on which point $a$ lies.

Then there is a projection map

$$
\pi: B l(0)_{\mathbb{C}^{2}} \rightarrow \mathbb{C}^{2}
$$

which is called the blowing-up map.

We denote $E:=\pi^{-1}(0)$ the exceptional divisor of $\pi$. $B l(0)_{\mathbb{C}^{2}}$ can also be defined in coordinates as follows

$$
B l(0)_{\mathbb{C}^{2}}=\{(x, y, u: v): x v=y u\} \subset \mathbb{C}^{2} \times P .
$$

here $(x, y) \in \mathbb{C}^{2}$ and $(u: v)$ are the homogeneous coordinates of $P$. Let

$$
\begin{aligned}
& V_{1}=\left\{(x, x v, 1: v): x, v \in \mathbb{C}^{2}\right\} \cong \mathbb{C}^{2} \\
& V_{2}=\left\{(y u, u, u: 1): y, u \in \mathbb{C}^{2}\right\} \cong \mathbb{C}^{2}
\end{aligned}
$$

then $V_{1} \cup V_{2}=B l(0)_{\mathbb{C}^{2}}$ is an affine covering.

2.2. Definition. Let $(V(f), 0)$ be a curve singularity. Then the closure of $\pi^{-1}(f \backslash 0)$ is called the strict transform of $f$, and the inverse image $\pi^{-1}(f)$ is called the total transform of $f$.

Let $f=\cup_{i=1}^{r} f_{i} \subset \mathbb{C}^{2}$ be a small representative of a reducible plane curve singularity with branches $f_{1}, \ldots, f_{r} \quad r \geq 1$. Assume that $X_{i} \stackrel{\pi_{i}}{\rightarrow} \ldots \stackrel{\pi_{2}}{\rightarrow} X_{1} \stackrel{\pi_{1}}{\rightarrow} \mathbb{C}^{2}$ is a sequence of blowing up points. Denote by $E^{(i)}=\left(\pi_{1} \circ \ldots \circ \pi_{i}\right)^{-1}(0)$ the exceptional divisor, $f^{(i)}=\overline{\left(\pi_{1} \circ \ldots \circ \pi_{i}\right)^{-1}(f \backslash\{0\})}$ the strict transform and $\left(\pi_{1} \circ \ldots \circ \pi_{i}\right)^{-1}(f)$ the total transform of $f$. Let $X_{i+1} \stackrel{\pi_{i+1}}{\rightarrow} X_{i}$ be the blowing up of $X_{i}$ in all points of $f^{(i)} \cap E^{(i)}$ which are still singular on $f^{(i)}$ or non-transversal intersection of $f^{(i)}$ with $E^{(i)}$ that is the points with intersection multiplicity of $f^{(i)}$ and $E^{(i)}$ greater than one or where two exceptional divisors and $f^{(i)}$ meet.

2.3. Definition. (i) $X_{k} \stackrel{\pi_{k}}{\rightarrow} \stackrel{\pi_{1}}{\rightarrow} X_{1} \rightarrow \mathbb{C}^{2}$ is called a standard resolution of $(V(f), 0)$ if all branches of $f^{(k)}$ are smooth, do not intersect each other, do intersect just one component of $E^{(k)}$ and do intersect this component transversally. 
We consider the following weighted graph, the resolution graph of $f$.

(i) To each component of $E^{(k)}$ a point $\bullet$ is associated.

(ii) To each component of $f^{(k)}$ a point $*$ is associated.

(iii) Two points are connected by an edge if the corresponding components intersect.

(iv) The points of type(i) are weighted. Let $E$ be a component of $E^{(k)}$. We give to the corresponding point the weight $i$ if $E$ is created in the $i-t h$ level of the blowing ups that is $i$ is minimal such that $\pi_{i+1} \circ \ldots \circ \pi_{n}(E)$ is not a point.

2.4. Definition. Let $n, m$ be positive integers then by the Euclidean algorithm, we can expand $\frac{n}{m}$ in a continued fraction in nonnegative integers $c_{i}$,

$$
\frac{n}{m}=c_{1}+\frac{1}{c_{2}+\frac{1}{c_{3}+\frac{1}{\ddots}+\frac{1}{c_{n}}}}
$$

We denote it by $\frac{n}{m}=\left[c_{1}, c_{2}, \ldots, c_{n}\right]$.

\section{Classification of Resolution Graphs of Weighted Homogeneous plane Curve Singularities}

The type of nondegenerate quasihomogeneous polynomials in two variables according to V.I. Arnold, S. M. Gusein-Zade and A. N. Varchenko [1] is given in the following table.

Table 1

\begin{tabular}{|c|c|c|}
\hline Type & Quasihomogeneous polynomial & Weighted Vector \\
\hline$I$ & $x^{a}+y^{b} \quad a, b \in \mathbb{Z}_{>0}$ & $(b, a, a b)$ \\
\hline$I I$ & $x^{a} y+y^{b} \quad a \in \mathbb{Z}_{>0}, b \in \mathbb{Z}_{>1}$ & $(b-1, a, a b)$ \\
\hline$I I I$ & $x^{a} y+y^{b} x \quad a, b \in \mathbb{Z}_{>1}$ & $(b-1, a-1, a b-1)$ \\
\hline
\end{tabular}

3.1. Proposition. Any weighted homogeneous polynomial $f \in \mathbb{C}[x, y]$ defining an isolated singularity is of the type $f=f_{0}+h$, where $f_{0}$ is one of the form given in the table. 1 and $h$ consist on terms having the same degree as $f_{0}$.

Proof. See [1].

3.2. Remark. The resolution of weighted homogeneous polynomial $f$ defining an isolated singularity does not depend on $h$ and depends only on weights and degree (see Theorem-3.5 in [6]).

3.3. Definition. $(V(f), 0) \subseteq\left(\mathbb{C}^{2}, 0\right)$ is called a quasihomogeneous plane curve singularity, if there exist an automorphism

$$
\phi: \mathbb{C}[[x, y]] \rightarrow \mathbb{C}[[x, y]]
$$

such that $\phi(f)$ is a weighted homogeneous plane curve singularity.

The following proposition gives us a combinatorial formula to compute the order and size of the resolution graph of a weighted homogeneous plane curve singularity. 
3.4. Proposition. Let $G_{f}$ denote the resolution graph of a plane curve singularity $(V(f), 0)$, where $f$ is weighted homogeneous polynomial defining an isolated singularity is of the type $f=f_{0}+h$, where $f_{0}$ is one of the form given in the table. 1 and $h$ consist on terms having the same degree as $f_{0}$. Then if

i: $f$ is of type-I then the order of $G_{f}$ is $\sum_{i=1}^{n} c_{i}+\operatorname{gcd}(a, b)$, where $\frac{b}{a}=\left[c_{1}, c_{2}, \ldots, c_{n}\right]$.

ii: $f$ is of type-II then the order of $G_{f}$ is $\sum_{i=1}^{n} c_{i}+\operatorname{gcd}(a, b-1)+1$, where $\frac{b-1}{a}=$ $\left[c_{1}, c_{2}, \ldots, c_{n}\right]$.

iii: $f$ is of type-III then the order of $G_{f}$ is $\sum_{i=1}^{n} c_{i}+\operatorname{gcd}(a-1, b-1)+2$, where $\frac{b-1}{a-1}=\left[c_{1}, c_{2}, \ldots, c_{n}\right]$.

Moreover $G_{f}$ is always a caterpillar.

Proof. $\quad$ i: If $(V(f), 0)$ be a weighted homogeneous plane curve singularity of typeI. Then it is noted that number of branches of the plane curve singularity is $\operatorname{gcd}(a, b)$ and resolution graphs $G_{f}$ and $G_{f_{0}}$ are same (see remark 3.2). We consider $a \leq b$. The case for $a>b$ can be treated in a similar way.

We start the resolution of singularity by the following blow up

$$
x \rightarrow x y, y \rightarrow y
$$

(This chart is only considered since the exceptional divisor does not intersect the curve in the other chart.)

Then we have the strict transformation $x^{a}+y^{b-a}=0$ and exceptional divisor $E_{1}$ : $y=0$. After $\left[\frac{b}{a}\right]=c_{1}$ blow ups we have the strict transformation $x^{a}+y^{b-c_{1} a}=0$ such that $b-c_{1} a<a$. Then multiplicity of strict transformation dropped and is equal to $b-c_{1} a$ and exceptional divisor $E_{c_{1}}: y=0$. Then make the blow up

$$
x \rightarrow x, y \rightarrow x y
$$

we get the strict transformation $x^{a-\left(b-c_{1} a\right)}+y^{b-c_{1} a}=0$ and exceptional divisor $E_{c_{1}+1}: x=0$. After $\left[\frac{a}{c}\right]=c_{2}$ blow ups we have the strict transformation $x^{a-c_{2}\left(b-c_{1} a\right)}+y^{b-c_{1} a}=0$ such that $a-c_{2}\left(b-c_{1} a\right)<b-c_{1} a$. Then multiplicity of strict transformation dropped and becomes equal to $a-c_{2}\left(b-c_{1} a\right)$ and exceptional divisor $E_{c_{1}+c_{2}}: y=0$. Continue in this way, after $c_{1}+c_{2}+\cdots+c_{n}$ blow ups we get the standard resolution. So the number of vertices of $G_{f}$ is $\sum_{i=1}^{n} c_{i}+\operatorname{gcd}(a, b)$ and if we construct the dual graph of this resolution as described in section- 2 then we find $G_{f}$ is a caterpillar.

ii and iii can be proved similarly to $\mathbf{i}$.

3.5. Remark. In the above proposition $\sum_{i=1}^{n} c_{i}$ is the number of dot vertices which represents the number of blow-ups required to make the standard resolution and $g c d(a, b)$ is the number of star vertices of the resolution graph which denote the number of branches of weighted homogeneous plane curve singularity of type-I.

3.6. Remark. If $\frac{n}{m}=\left[c_{1}, c_{2}, \ldots, c_{n}\right]$ then in the following proposition, the integers $e_{i}$, $a_{t}$ and $b_{s}$ denote the sum $\sum_{k=1}^{i} c_{k}$, the weight of the vertex $v_{t}$ and the weight of the vertex $v_{s}$ respectively.

In the following proposition we describe a combinatorial construction to compute the resolution graph of a weighted homogeneous plane curve singularity of type-I. 
3.7. Proposition. Let $(V(f), 0)$ be a weighted homogeneous plane curve singularity of type-I then its resolution grpah $G_{f}$ can be obtained by using the following construction.

Proof. Since $(V(f), 0)$ be a weighted homogeneous plane curve singularity of type-I, then from Proposition-3.4 we have the set of vertices

$$
V=\left\{v_{i}: 1 \leq i \leq \sum_{i=1}^{n} c_{i}+\operatorname{gcd}(a, b)\right\},
$$

where $\frac{b}{a}=\left[c_{1}, c_{2}, \ldots, c_{n}\right]$. Then we can define the integers such that $e_{1}<e_{2}<\cdots<e_{n}$, where $e_{i}=\sum_{k=1}^{i} c_{k}$ and a partion on the set of vertices $V$ such that $V=V_{\bullet}^{(1)} \cup V_{\bullet}^{(2)} \cup$ $V_{\bullet}^{(3)} \cup V_{*}$, where

$$
\begin{gathered}
V_{\bullet}^{(1)}=\left\{v_{i}: 1 \leq i \leq e_{1}\right\}, \\
V_{\bullet}^{(2)}=\left\{v_{i}: e_{1}+1 \leq i \leq e_{1}+l\right\}, \\
V_{\bullet}^{(3)}=\left\{v_{i}: e_{1}+l+1 \leq i \leq e_{n}\right\},
\end{gathered}
$$

where

$$
l= \begin{cases}\frac{\sum_{j=1}^{2}}{2}\left(e_{2 j+1}-e_{2 j}\right), & \text { if } \mathrm{n} \text { is even; } \\ \frac{n-1}{\sum_{j=1}^{2}}\left(e_{2 j+1}-e_{2 j}\right), & \text { if } \mathrm{n} \text { is odd; }\end{cases}
$$

$$
V_{*}=\left\{v_{i}^{*}: 1 \leq i \leq \operatorname{gcd}(a, b)\right\} .
$$

Now define

$$
A:=\left\{e_{2}+1, \ldots, e_{3}, e_{4}+1, \ldots, e_{5}, \ldots, e_{2\left[\frac{n-1}{2}\right]}+1, \ldots, e_{2\left[\frac{n-1}{2}\right]+1}\right\}=\left\{a_{e_{1}+1}, \ldots, a_{e_{1}+l}\right\}
$$

and

$B:=\left\{e_{1}+1, \ldots, e_{2}, e_{3}+1, \ldots, e_{4}, \ldots, e_{2\left[\frac{n-1}{2}\right]+1}+1, \ldots, e_{2\left[\frac{n}{2}\right]}\right\}=\left\{b_{e_{n}}, b_{e_{n}-1}, \ldots, b_{e_{1}+l+1}\right\}$.

Note that $\left|V_{\bullet}^{(2)}\right|=|A|$ and $\left|V_{\bullet}^{(3)}\right|=|B|$.

Let

$$
s= \begin{cases}1, & \text { if } \mathrm{n} \text { is even; } \\ 0, & \text { if } \mathrm{n} \text { is odd; }\end{cases}
$$

and $q=e_{1}+l+1+s$ then we obtain the following resolution graph

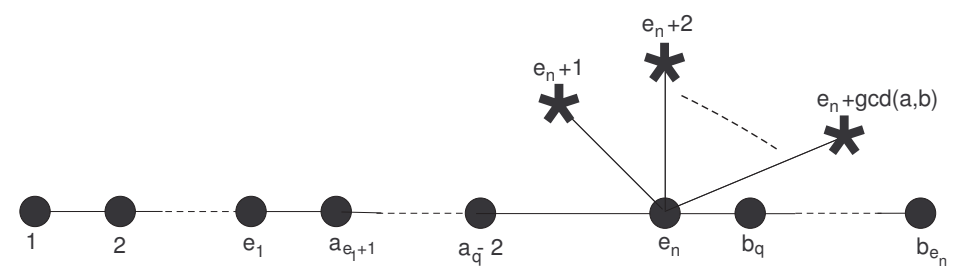


3.8. Example. Consider $(V(f), 0)$ be a weighted homogeneous plane curve singularity with weights $(230,1055)$.

Then $V=\left\{v_{1}, v_{2}, \ldots, v_{13}, v_{1}^{*}, v_{2}^{*}, v_{3}^{*}, v_{4}^{*}, v_{5}^{*}\right\}$ (see Proposition-3.4). Now by using the Proposition-3.7 we can construct the following data:

Step - 1 : Construct

$$
e_{1}=4, e_{2}=5, e_{3}=6, e_{4}=8, e_{5}=10, e_{6}=11, e_{7}=13 .
$$

Step -2 : Since $n=7$ which is odd therefore $V=V_{\bullet}^{(1)} \cup V_{\bullet}^{(2)} \cup V_{\bullet}^{(3)} \cup V_{*}$, where

$$
\begin{gathered}
V_{\bullet}^{(1)}=\left\{v_{1}, v_{2}, v_{3}, v_{4}\right\}, \\
V_{\bullet}^{(2)}=\left\{v_{5}, v_{6}, v_{7}, v_{8}, v_{9}\right\}, \\
V_{\bullet}^{(3)}=\left\{v_{10}, v_{11}, v_{12}, v_{13}\right\},
\end{gathered}
$$

and

Step -3 :

$$
V_{*}=\left\{v_{1}^{*}, v_{2}^{*}, v_{3}^{*}, v_{4}^{*}, v_{5}^{*}\right\} .
$$

$$
A=\{6,9,10,12,13\}=\left\{a_{5}, a_{6}, a_{7}, a_{8}, a_{9}\right\}
$$

nd

$$
B=\{5,7,8,11\}=\left\{b_{13}, b_{12}, b_{11}, b_{10}\right\} .
$$

Step $-4:$ Attach all $*$ vertices with $v_{9}$ then we get the resolution graph as given in Figure 1.

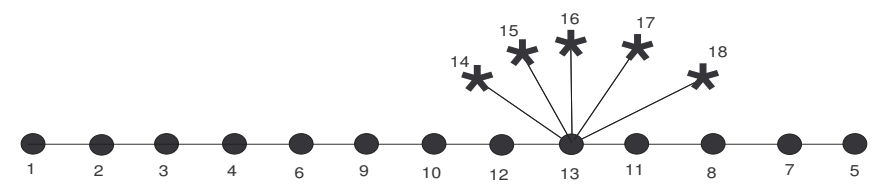

Figure 1. Resolution graph of a weighted homogeneous plane curve singularity with weights $(230,1055)$.

In the following two propositions we describe a combinatorial construction to compute the resolution graph of weighted homogeneous plane curve singularities of type II and III.

3.9. Proposition. Let $(V(f), 0)$ be a weighted homogeneous plane curve singularity of type-II then its resolution graph $G_{f}$ is one of the graphs given in Table 2.

Proof. Since $(V(f), 0)$ be a weighted homogeneous plane curve singularity of type-II, then we have

$$
V=\left\{v_{i}: 1 \leq i \leq \sum_{i=1}^{n} c_{i}+(\operatorname{gcd}(a, b-1)+1)\right\},
$$

Make a partition of $V$ such that $V=V^{\prime} \cup V_{*}^{(1)}$, where

$$
V^{\prime}=\left\{v_{i}: 1 \leq i \leq \sum_{i=1}^{n} c_{i}+\operatorname{gcd}(a, b-1)\right\}
$$

and

$$
V_{*}^{(1)}=\left\{v_{i}^{*}: i=\operatorname{gcd}(a, b-1)+1\right\} .
$$

For $V^{\prime}$ follow the construction as explained in Proposition-3.7. Assign the weight $e_{n}+\operatorname{gcd}(a, b-1)+1$ to the $*$ vertex $v_{i}^{*}$ for $i=\operatorname{gcd}(a, b-1)+1$. If $a \leq b-1$ then attach 
Table 2

\begin{tabular}{|c|c|c|}
\hline Type & Condition & Resolution Graph \\
\hline . & 西 & 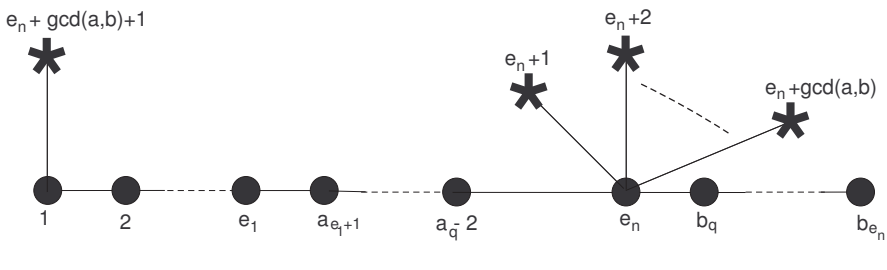 \\
\hline$I I$ & 更 & $\underbrace{\cdots}_{b_{q}} \star^{e_{n}+g c d(a, b)} \underbrace{e_{n}+\operatorname{gcd}(a, b)+1}_{b_{e_{n}}}$ \\
\hline
\end{tabular}

$v_{i}^{*}$ with the $\bullet$ vertex of weight 1 . If $a>b-1$ then attach $v_{i}^{*}$ with the vertex of $V_{\bullet}^{\prime(3)}$ with smallest weight.

3.10. Proposition. Let $(V(f), 0)$ be a weighted homogeneous plane curve singularity of type-III then its resolution grpah $G_{f}$ is the following:

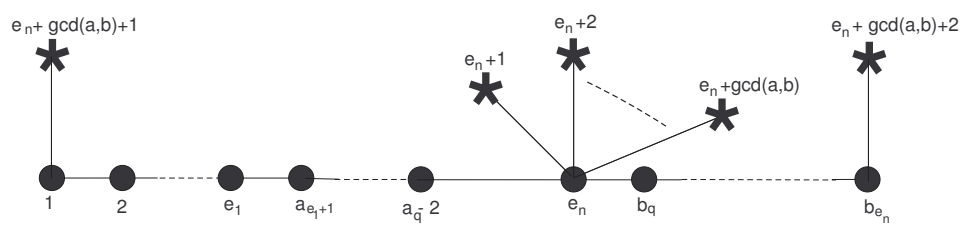

Figure 2. Resolution graph of $(V(f), 0)$

Proof. Since $(V(f), 0)$ be a weighted homogeneous plane curve singularity of type-III, then we have

$$
V=\left\{v_{i}: 1 \leq i \leq \sum_{i=1}^{n} c_{i}+(\operatorname{gcd}(a-1, b-1)+2)\right\},
$$

Make a partition of $V$ such that $V=V^{\prime} \cup V_{*}^{(1)}$, where

and

$$
V^{\prime}=\left\{v_{i}: 1 \leq i \leq \sum_{i=1}^{n} c_{i}+\operatorname{gcd}(a-1, b-1)\right\}
$$

$$
V_{*}^{(1)}=\left\{v_{i}^{*}: i=\operatorname{gcd}(a-1, b-1)+1, \operatorname{gcd}(a-1, b-1)+2\right\},
$$

For $V^{\prime}$ follow the construction as explained in Proposition-3.7. Assign the weights $e_{n}+\operatorname{gcd}(a-1, b-1)+1, e_{n}+\operatorname{gcd}(a-1, b-1)+2$, to the $*$ vertices $v_{i}^{*}$ for $i=\operatorname{gcd}(a-1, b-1)+1$ and $i=\operatorname{gcd}(a-1, b-1)+2$ respectively. Attach $v_{i}^{*}$ for $i=\operatorname{gcd}(a-1, b-1)+1$ with the - vertex of weight 1 and attach $v_{i}^{*}$ for $i=\operatorname{gcd}(a-1, b-1)+2$ with the $\bullet$ vertex of $V_{\bullet}^{\prime}(3)$ with smallest weight. 


\section{Acknowledgements}

The authors would like to thank the referee for his/her corrections, comments and useful criticism which improved the first version of this paper.

\section{References}

[1] Arnold, V. I.; Gusein-Zade, S. M.; Varchenko,A. N. Singularities of Differentiable Maps, Volume I, Birkhäuser, Boston Basel Berlin (1985).

[2] Cutkosky, S. D. and Srinivasan, H.; The algebraic fundamental group of a curve singularity, Journal of Algebra 230, 101-126, (2000).

[3] De Jong, T. and Pfister, G.; Local Analytic Geometry, Vieweg (2000).

[4] Jingen, Y.; Curve Singularities and Graphs, Acta Mathematica Sinica, 6 (1), 87-96, (1990).

[5] Kollár, J.; Lectures on Resolution of Singularities, Princeton University Press (2007).

[6] Kang, C.; Analytic Types of Plane Curve Singularities defined by Weighted Homogeneous Polynomials, Trans. A.M.S. 352 (9), 3995-4006, (2000).

[7] Muhly, H.T. and Zariski, O.; The Resolution of Singularities of an Algebraic curve, Amer.J.Math., 61 (1), 107-114, (1939).

[8] Saito, K.; Quasihomogene isolierte singularitäten von hyperflächen, Invent. Math. 14, 123142, (1971).

[9] Wall, C.T.C.; Singular Points of Plane Curves, Cambridge University Press (2004). 